\title{
Clinical analysis of 50 patients with heterotopic pregnancy after ovulation induction or embryo transfer
}

\author{
Zaigui Wu, Xinmei Zhang, Ping Xu and Xiufeng Huang ${ }^{*}$
}

\begin{abstract}
Objectives: The purpose of this study was to evaluate the clinical characteristics, prenatal diagnosis, and management of patients with heterotopic pregnancy after ovulation induction or embryo transfer.

Methods: This was a retrospective study of fifty cases with heterotopic pregnancy, in which the fertilization way, type and number of embryo transferred, gestational age, clinical presentation and outcome of intrauterine, ultrasound presentation and site of ectopic pregnancy, bilateral fallopian tube and treatment were evaluated.

Results: Six patients had spontaneous pregnancy and two had artificial insemination after ovulation induction. Sixteen had fresh and nineteen had frozen embryo transfer with seven patients unrecorded its embryo type and number. The average days from transplantation (or intercourse/insemination) to diagnosing heterotopic pregnancy was thirty-seven with the earliest eighteen and the latest more than 70 days. Although the most common presentation was vaginal bleeding or abdominal pain, more than $21 \%$ was found by ultrasound and rare individuals even presented with gastrointestinal symptom which may imply ruptured EP and hemorrhagic shock. Giving proper treatment (surgery or local drug injection), the majority of them had a successful intrauterine pregnancy with only seven miscarried.

Conclusions: Ovulation induction or embryo transfer increased the risk of HP greatly and clinician should raise high suspicious during the whole first trimester. Although the most ectopic site was ampullary, other sites such as cornual, cervical, abdominal especially interstitial or tubal stumps should also be assessed by ultrasound even in patients with bilateral salpingectomy or tubal obstructed. Repeated ultrasound tests 2 weeks after the diagnosis of intrauterine pregnancy with heart beating was very necessary to find the missed ones in suspicious patients. Compared with surgery, embryo suction with or without proper local drug injection would be more advisable for patients with cervical, cornual, or interstitial pregnancy in order to reserve the intrauterine pregnancy.
\end{abstract}

Keywords: Heterotopic pregnancy, Ectopic pregnancy, Controlled ovarian hyperstimulation

\section{Background}

Heterotopic pregnancy (HP), first reported in 1708, was defined as the co-incidence of intrauterine and ectopic pregnancy. That is, the embryos implanted at two or more different sites simultaneously. In natural cycles, usually only one follicle developed and ovulated, the spontaneous incidence of HP was extremely low (one

*Correspondence: huangxiufeng73@163.com; huangxiufeng@zju.edu.cn Women's Hospital, School of Medicine, Zhejiang University, Hangzhou, China in 30,000 pregnancies) [1]. Due to the extended use of assisted reproductive technologies (ARTs), the incidence of HP had been reported to be up to $1-11 \%$ [2]. Therefore, early diagnosis and proper treatments were very important for them to remove the ectopic pregnancy (EP) while reserve the intrauterine pregnancy (IUP).

In clinic, it was often difficult to diagnose the HP as early as possible because large amount of them were asymptomatic or just masked by enlarged ovaries after ovulation induction $[3,4]$. The most common EP site was fallopian tubal, but other sites such as cornual, cervical,

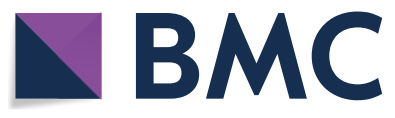

(c) The Author(s) 2018. This article is distributed under the terms of the Creative Commons Attribution 4.0 International License (http://creativecommons.org/licenses/by/4.0/), which permits unrestricted use, distribution, and reproduction in any medium, provided you give appropriate credit to the original author(s) and the source, provide a link to the Creative Commons license, and indicate if changes were made. The Creative Commons Public Domain Dedication waiver (http://creativecommons.org/ publicdomain/zero/1.0/) applies to the data made available in this article, unless otherwise stated. 
abdominal, interstitial, or tubal stumps pregnancy all had been reported in the literature [5] and our paper. Thus, the clinician and sonographer should raise high suspicious of HP with patients transplanting multiple embryos or having ovulation induction.

Once HP was diagnosed, the optimal solution should be carried out. The choice of therapeutic approach relied on many factors, of which the vital one is to preserve the coexisting IUP as possible. First, systematic methotrexate or embolization was not suitable for HP patients unless the IUP aborted. Second, the traditional salpingectomy may be still practicable to HP patients with tubal pregnancy, but for special types such as cornual and interstitial pregnancy the conventional management with salpingectomy and cornual resection should no longer be the first choice. The alternative method would be embryo suction combined with local injection of MTX or $10 \%$ KCL [6]. It, to a certain extent, could relieve the risk of uterine rupture during the subsequent pregnancy.

The purpose of this paper was to review all patients who had HP at our center from 2007 to 2015 to make the clinician impressed on the diagnosis and treatment of HP.

\section{Patients and methods}

This retrospective study included all the patients diagnosed with HP from January 2007 to September 2015 at the study center. In our center, the first ultrasound test was carried out 4-5 weeks after ET in general and a repeated test was also performed 2 weeks after the diagnosed of an IUP with heart beaten. If patients had vaginal bleeding or abdominal pain ultrasound test would be taken at any time. Fertilization way, gestational age at diagnosis, the ultrasound characteristics of IUP and EP, clinical symptoms, bilateral fallopian tubes, EP site, and treatments were recorded and analyzed; the results are described in Tables 1, 2, 3, 4, and 5 .

\section{Results}

A total of fifty patients were identified with HP (Tables 1, 2, 3, 4 and 5). Coexisting with the IUP, forty-two patients had conceived with IVF-ET, two with intrauterine insemination (IUI) and the other six conceived naturally after ovulation induction. The average time being diagnosed was 37 days after transplantation (or insemination/intercourse) with the shortest 18 days and the longest more than 9 weeks (CRL $4.7 \mathrm{~cm}$ ). Thirty-two patients had clinical manifestation (fifteen only with vaginal bleeding, eight only with abdominal pain while nine with both), one with hemorrhagic shock due to the EP rupture, another two only with vomiting while the remaining fifteen were asymptomatic.

All patients were diagnosed by ultrasound among whom the visualization of heart activity in both intrauterine and extrauterine gestations was observed in twenty patients. There were twenty-five patients with heart activity seen only in IUP (four had a visible gestational sac in extrauterine gestations while the other twenty-one possessed an adnexal mass). Seven patients were diagnosed with IUP miscarriage (of the extrauterine pregnancy, three with and two without heart activity, two with an adnexal mass) based on the inability to see an embryonic pole or cardiac activity between 6 and 10 weeks. In five patients, transvaginal ultrasound revealed an intrauterine pregnancy with heart beaten appropriate for seven to 8 weeks, gestation while no suggestive findings of an ectopic pregnancy were demonstrated and they were eventually diagnosed with HP two to 3 weeks later.

The most common HP type was IUP complicated with tubal pregnancy including twenty-seven ampullary EP, one isthmic EP, eleven interstitial EP and three tubal stumps pregnancies. Of the rest of eight cases, five were cornual, once was cervical, and two were abdominal. Depending on the location of the ectopic pregnancy, the method of transvaginal ultrasound-guided suction aspiration with or without intracardiac MTX or potassium chloride (KCL) injection had been carried out in five interstitial EP, two cornual EP, one tubal stumps pregnancy, and one cervical EP patients. Due to the rupture of EP, the tubal stumps pregnancy and one interstitial pregnancy patients had the emergency surgery 1 or 2 weeks after the above treatment and another interstitial pregnancy patient had the same experience for enlargement of the adnexal mass 3 weeks later.

As to bilateral fallopian tubes, eleven patients had prior ipsilateral salpingectomy for tubal pregnancy with four interstitial EP, four cornual EP, and three tubal stumps pregnancy this time. Two earlier experienced ipsilateral salpingostomy, and the other five had a history of contralateral tubal pregnancy. A ruptured abdominal pregnancy was observed after bilateral salpingectomy for tubal pregnancy while two interstitial and three ampullary pregnancy patients proved to be the tubal obstruction by hysterosalpingography (HSG) or laparoscopic surgery. Thirteen patients had a history of bilateral salpingitis and the remaining twelve had normal tubes.

Tables 1, 2, 3, 4, and 5 show clinical data of fifty patients with HP in the present study.

\section{Discussion}

It was established that the assisted reproduction techniques (ART) had significantly increased the incidence of HP [7] which could also be showed from our center previous study [5] and this report. According to its definition, we could easily understand ovulation induction or transferring multiple embryo was just the first step to 


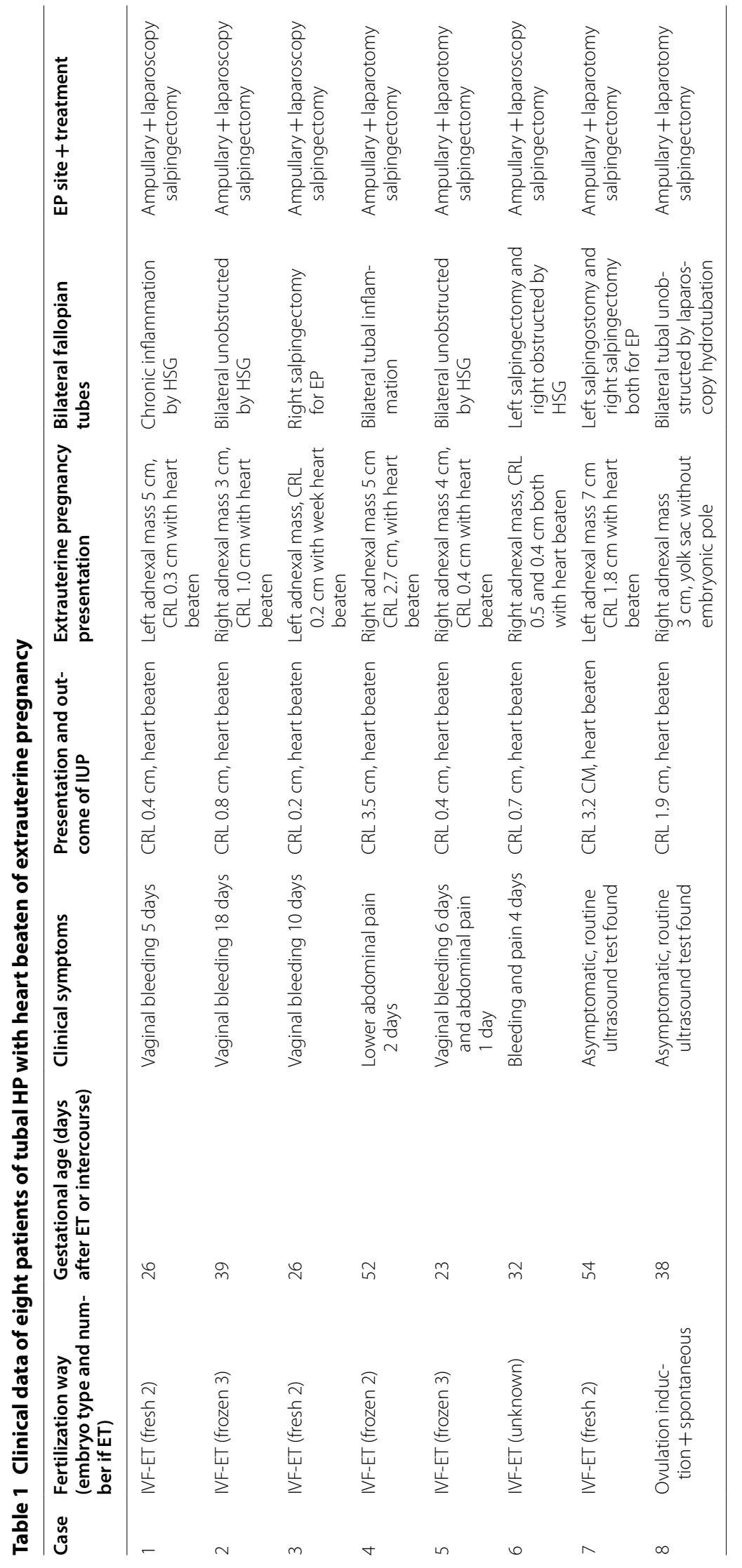




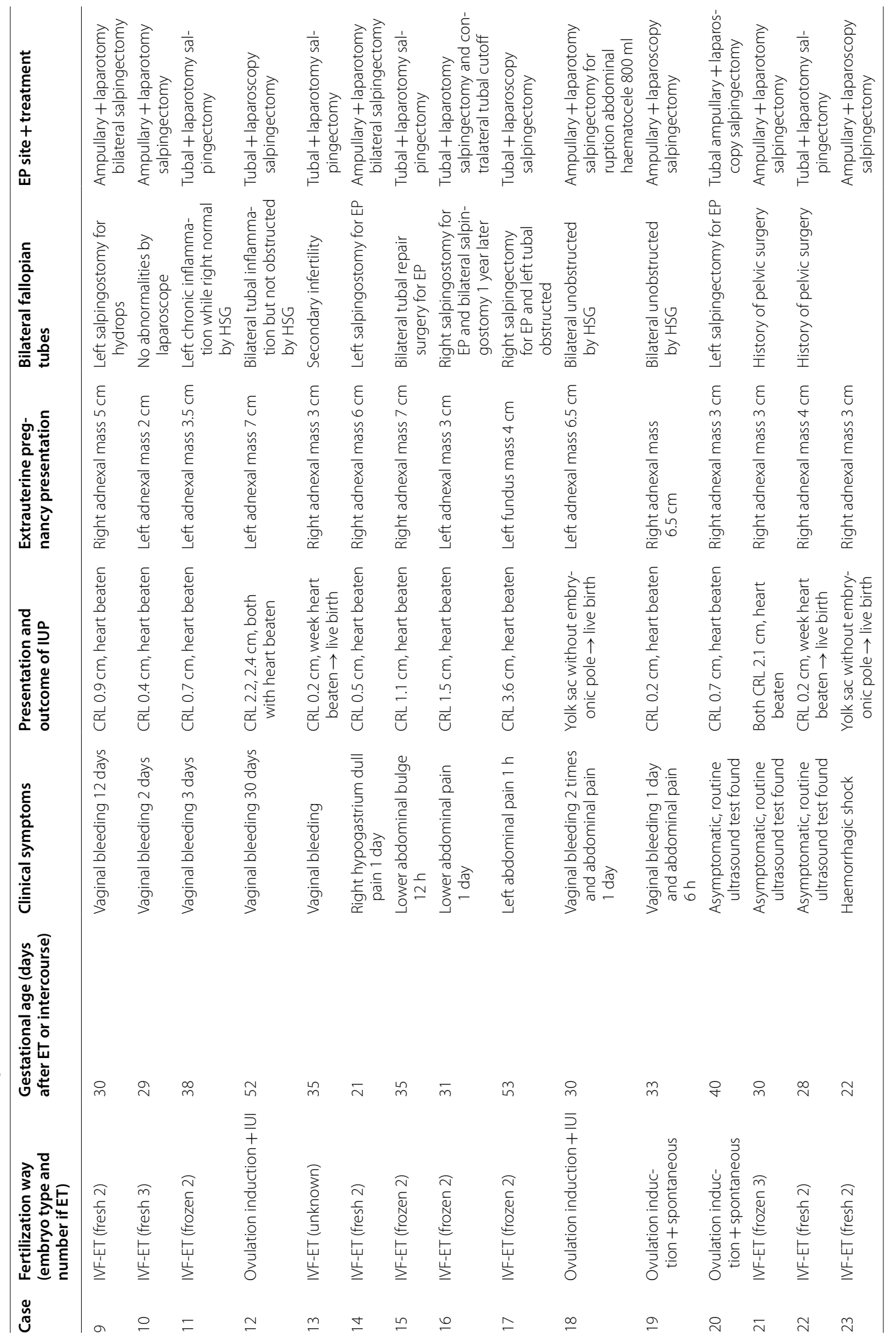




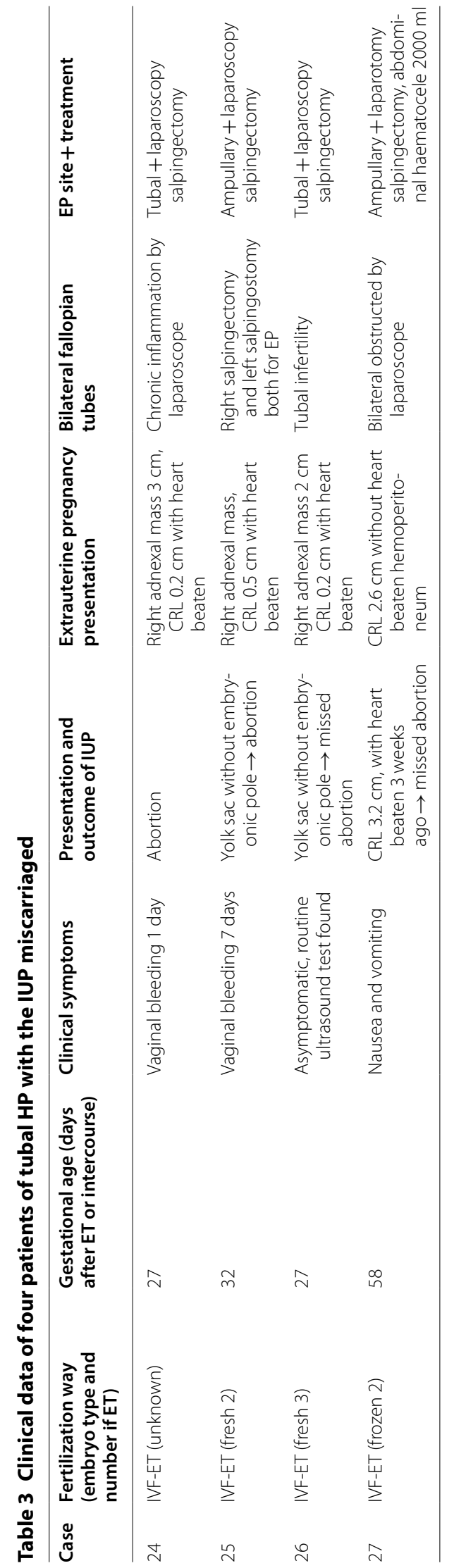




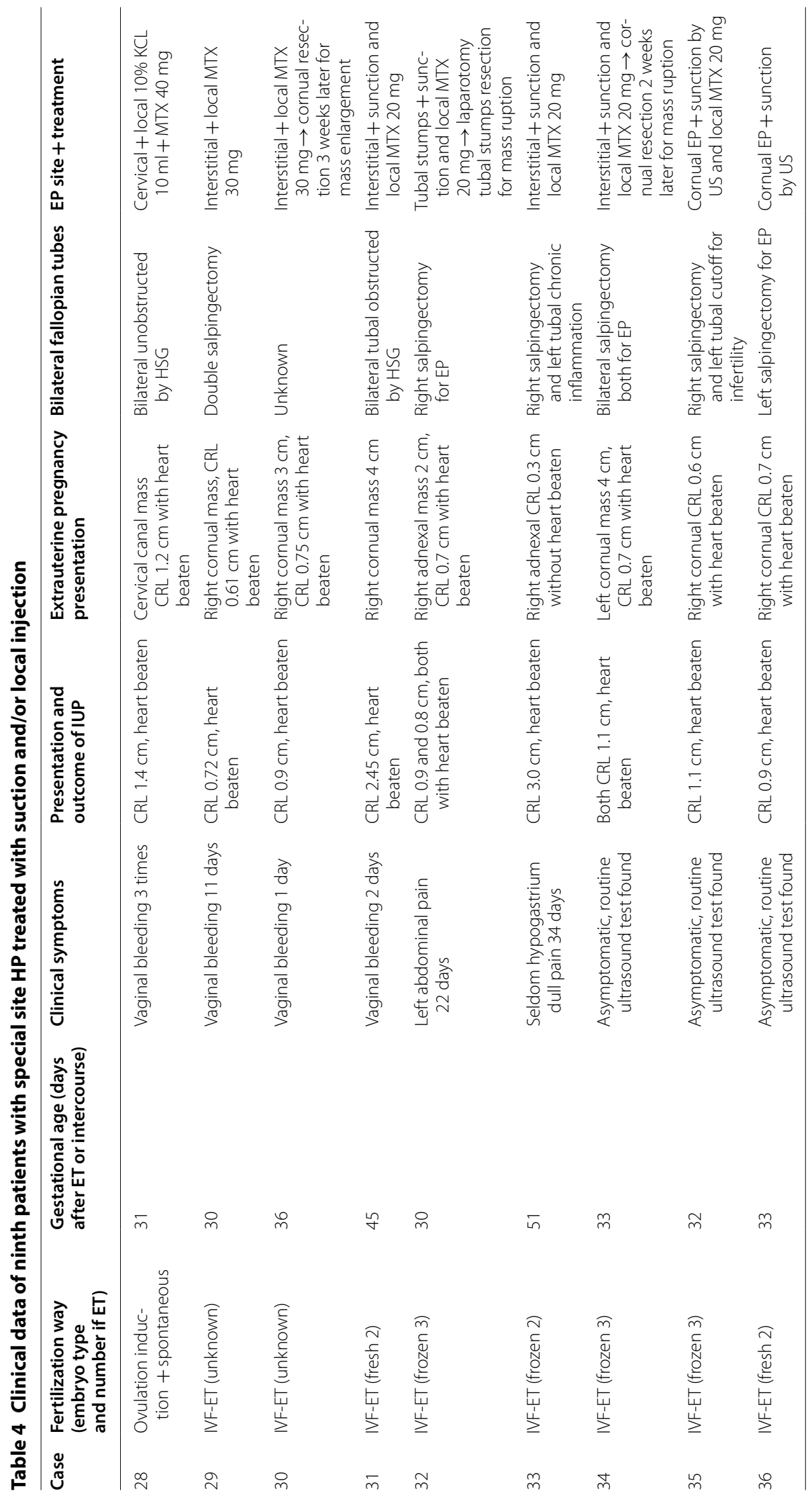




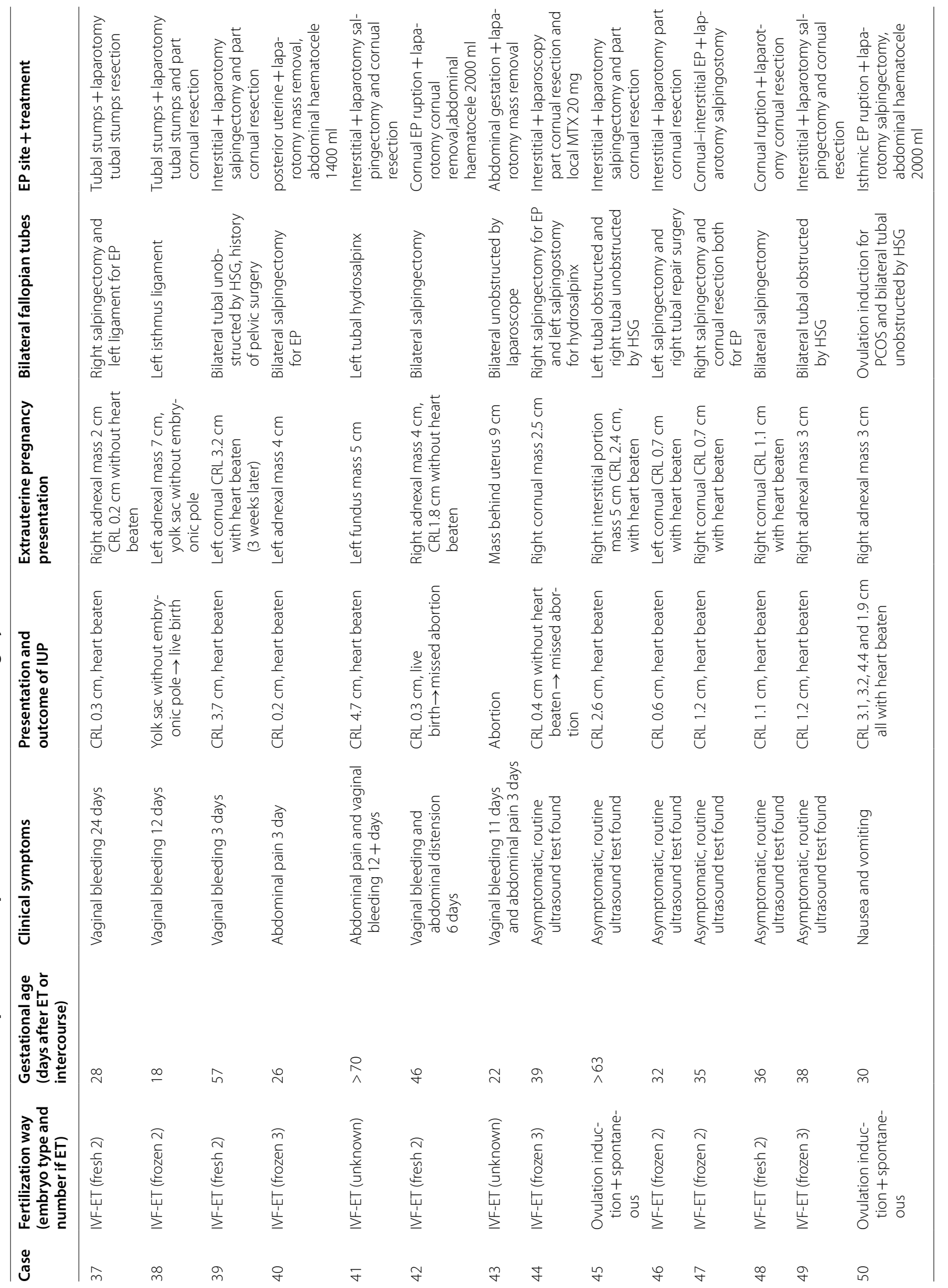


make multi-site implantation possible. There were many other risk factors predisposing the development of HP and we suggested that tubal malfunction worked vitally.

Tubal pregnancies were the most common type of EP with HP as described in our study with twenty-six ampullary in fifty HP patients. The recurrent rate of EP ranges from 15 to $20 \%$ in condition to one previous EP treated by linear salpingostomy and it increases to $32 \%$ in patients with two [8]. Of the fifty HP patients, fifteen had a history of previous EP with the most three times. The exact pathomechanism was unclear while the latest literature $[9,10]$ suggested tubal malfunction had a predominant role in the pathogenesis of tubal implantation altering the tubal transport mechanisms and expression of molecules normally inhibiting tubal implantation. It was just the alteration of transport mechanisms and molecules expression that explained why ipsilateral salpingectomy, in clinical, could not totally prevent the recurrent EP but to increase the occurrence of cornual-interstitial pregnancy. In our present study, a total of twenty patients had ipsilateral salpingectomy or even bilateral salpingectomy but this time five had cornual, four had interstitial, and two had tubal stump pregnancy. Moreover, abdominal EP may even be happened on the condition of bilateral salpingectomy which has been reported only four cases with two of ours [11-13]. All together suggested that the risk factors may alter the transport mechanisms and molecules expression systemically. Thus when having ultrasound test, not only the fallopian tubes but also the cervix, previous cesarean scar, cornual region, ovaries, and even abdominal cavity should all be examined carefully regardless of bilateral salpingectomy or tubal obstruction.

As HP was relatively rare and clinical features were nonspecific, the misdiagnosis or delayed diagnosis occurred very frequently $[7,14]$. We could see that a great number of patients were asymptomatic (29\% in our study and $50 \%$ in the literature [15]) or just presented with little vaginal bleeding and/or abdominal pain which could be mistaken for threatened abortion of IUP. Gastrointestinal symptoms such as vomiting or diarrhea can also be seen at times and even some patients experienced shock directly due to the rupture of EP [14]. Careful transvaginal ultrasound evaluation should be performed with these symptomatic patients at any time as the earliest gestational age at ultrasound diagnosis of HP in our center was only 18 days after ET. But for those asymptomatic patients, the first transvaginal ultrasound evaluation can be carried out at 4-5 weeks after ET (or 6-7 gestational weeks) as reported in our study [16]. When the diagnosis of HP was made, a gestational sac was visible in more than half of the extrauterine pregnancies and a heart beaten was even seen in $44 \%$ of these patients with the others possessing an adnexal mass. But ten patients (20\%) were diagnosed with HP at more than 9 weeks and four of them even had an intrauterine pregnancy appropriate for more than 7 weeks' gestation while no ultrasonic findings were detected to imply an ectopic pregnancy on her initial ultrasonic testing. Because patients treated with ovulation or ET usually had exact fertilization date, the embryonic development of intrauterine and extrauterine estimated by crown-rump length (CRL) differed no more than 1 week and it was certain that the diagnosis was delayed. As noted previously, above two-thirds patients require two or more ultrasound examinations to establish a diagnosis of HP, and repeated ultrasonic testing 2 weeks after the initial diagnosis of live IUP was very necessary to exclude the diagnosis of HP.

When diagnosed and treated timely and properly, the survival rate of the IUP in heterotopic pregnancies reached to $70 \%$ [17]. A large majority of $\mathrm{HP}(31 / 50)$ cases were diagnosed before 35 days after fertilization or ET and only seven patients miscarriaged eventually with the IUP. The treatment options include surgical, medical, and expectant therapy. Seven cases diagnosed with suspicious HP for a adnexal mass less than $4 \mathrm{~cm}$ were managed by expectant therapy successfully and they were not reported in our present study. Surgical management was performed in the overwhelming majority of HP cases as reported $78-90.78 \%$ in the literature $[4,18]$ and $81.63 \%$ in our report. Regarding ampullary and isthmic EP, salpingectomy via laparoscopy or laparotomy would not increase uterin rupture risk in subsequent pregnancy. But for special local EP such as cornual or interstitial EP and especially cervical pregnancy, surgery would increase the risk of IUP abortion or uterine rupture in future pregnancy. Medical treatment would provide an effective choice for these patients [19]. It consisted of suction aspiration or a combination with injections of MTX, KCL in the gestational sac or fetal heart [20]. The use of MTX was contraindicated previously because of its potential embryotoxicity on the IUP but recent studies have proved its safety and effectiveness with local injection of low-dose [21]. Four interstitial, two cornual, and one tubal stumps pregnancy were managed with sonographically guided suction aspiration and MTX injection alone while a combination of MTX and KCL was applied in the cervical pregnancy patients. Two interstitial and one tubal stumps pregnancy were eventually had surgery due to the rupture of EP in the following-up 1-3 weeks after the procedure. So patients choosing the medical treatment should be informed the risk of EP rupture and repeated ultrasound testing should be followed weekly. Once any clinical suspicion of ectopic pregnancy rupture occurs, a diagnostic laparoscopy or laparotomy must be done immediately. 
In conclusion, data from our study with fifty cases showed that any patients concepted by ovulation induction or ET could have the possibility of HP in spite of bilateral salpingectomy or tubal obstruction. Careful and repeated ultrasound testings should be carried out until the diagnosis of HP was excluded during the whole first trimester. Suction and local medical treatment was a feasible option for selected patients with special types of HP especially cornual, interstitial or cervical pregnancy.

\section{Authors' contributions}

WZ collected the clinical data, analyzed these data, and drafted the manuscript. HX helped to draft the manuscript and revised the manuscript. All authors read and approved the final manuscript.

\section{Acknowledgements}

The paper was supported by two funds: Science and Technology Fund of Zhejiang Province (2013C33149) and Zhejiang Key Medical Science Project (Minimally invasive Gynecology).

\section{Competing interests}

The authors declare that they have no competing interests.

\section{Consent for publication}

Written informed consent was obtained from the patient for publication of this case report and any accompanying images.

\section{Ethics approval and consent to participate}

This study was approved by the ethics committee of the Women's Hospital, School of Medicine Zhejiang University(No 2015099).

\section{Publisher's Note}

Springer Nature remains neutral with regard to jurisdictional claims in published maps and institutional affiliations.

Received: 26 April 2016 Accepted: 6 April 2018

Published online: 16 April 2018

\section{References}

1. Anastasakis E, Jetti A, Macara L, Daskalakis G. A case of heterotopic pregnancy in the absence of risk factors. A brief literature review. Fetal Diagn Ther. 2007;22:285-8.

2. Brunette $\mathrm{DD}$, Roline $\mathrm{C}$. Heterotopic pregnancy resulting from in vitro fertilization. Am J Emerg Med. 2011;29:960-1.

3. Ivkosic IE, Bauman R, Ujevic B, Vasilj O, Kojic IP, Ivkosic A, Vukusic D, Turudic TP. Spontaneous heterotopic, ectopic cervical and ectopic tubal pregnancy - ever present diagnostic difficulty: three case reports. Acta Clin Croat. 2015;54:367-70.

4. Barrenetxea G, Barinaga-Rementeria L, Lopez DLA, Agirregoikoa JA, Mandiola M, Carbonero K. Heterotopic pregnancy: two cases and a comparative review. Fertil Steril. 2007;87:417-9.

5. Yu Y, Xu W, Xie Z, Huang Q, Li S. Management and outcome of 25 heterotopic pregnancies in Zhejiang, China. Eur J Obstet Gynecol Reprod Biol. 2014:180:157-61.

6. Kumar R, Dey M. Spontaneous heterotopic pregnancy with tubal rupture and pregnancy progressing to term. Med J Armed Forces India. 2015;71:S73-5.

7. Ko JK, Cheung VY. A 12-year experience of the management and outcome of heterotopic pregnancy at Queen Mary Hospital, Hong Kong, China. Int J Gynaecol Obstet. 2012;119:194-5.

8. Moini A, Hosseini R, Jahangiri N, Shiva M, Akhoond MR. Risk factors for ectopic pregnancy: a case-control study. J Res Med Sci. 2014;19:844-9.
9. Refaat B, Simpson H, Britton E, Biswas J, Wells M, Aplin JD, Ledger W. Why does the fallopian tube fail in ectopic pregnancy? The role of activins, inducible nitric oxide synthase, and MUC1 in ectopic implantation. Fertil Steril. 2012;97:1115-23.

10. Refaat B. Role of activins in embryo implantation and diagnosis of ectopic pregnancy: a review. Reprod Biol Endocrinol. 2014;12:116.

11. Cormio G, Santamato S, Putignano G, Bettocchi S, Pascazio F. Concomitant abdominal and intrauterine pregnancy after in vitro fertilization in a woman with bilateral salpingectomy. A case report. J Reprod Med. 2003;48:747-9

12. Zhaoxia L, Honglang Q Danqing C. Ruptured heterotopic pregnancy after assisted reproduction in a patient who underwent bilateral salpingectomy. J Obstet Gynaecol. 2013;33:209-10.

13. Shavit T, Paz-Shalom E, Lachman E, Fainaru O, Ellenbogen A. Unusual case of recurrent heterotopic pregnancy after bilateral salpingectomy and literature review. Reprod Biomed Online. 2013;26:59-61.

14. Wang LL, Chen X, Ye DS, Liu YD, He YX, Guo W, Chen SL. Misdiagnosis and delayed diagnosis for ectopic and heterotopic pregnancies after in vitro fertilization and embryo transfer. J Huazhong Univ Sci Technol Med Sci. 2014;34:103-7.

15. Fernandez $H$, Lelaidier C, Doumerc S, Fournet P, Olivennes F, Frydman R. Nonsurgical treatment of heterotopic pregnancy: a report of six cases. Fertil Steril. 1993;60:428-32

16. Sentilhes $L$, Bouet $P E$, Jalle T, Boussion F, Lefebvre-Lacoeuille $C$, Descamps P. Ultrasound diagnosis of spontaneous bilateral tubal pregnancy. Aust N Z J Obstet Gynaecol. 2009;49:695-6.

17. Clayton HB, Schieve LA, Peterson HB, Jamieson DJ, Reynolds MA, Wright VC. A comparison of heterotopic and intrauterine-only pregnancy outcomes after assisted reproductive technologies in the United States from 1999 to 2002. Fertil Steril. 2007;87:303-9.

18. Diallo D, Aubard Y, Piver P, Baudet JH. Heterotopic pregnancy: a report of 5 cases and review of the literature. J Gynecol Obstet Biol Reprod (Paris). 2000:29:131-41.

19. Baxi A, Kaushal M, Karmalkar H, Sahu P, Kadhi P, Daval B. Successful expectant management of tubal heterotopic pregnancy. J Hum Reprod Sci. 2010;3:108-10.

20. Deka D, Bahadur A, Singh A, Malhotra N. Successful management of heterotopic pregnancy after fetal reduction using potassium chloride and methotrexate. J Hum Reprod Sci. 2012;5:57-60.

21. Sijanovic S, Vidosavljevic D, Sijanovic I. Methotrexate in local treatment of cervical heterotopic pregnancy with successful perinatal outcome: case report. J Obstet Gynaecol Res. 2011:37:1241-5.

Ready to submit your research? Choose BMC and benefit from

- fast, convenient online submission

- thorough peer review by experienced researchers in your field

- rapid publication on acceptance

- support for research data, including large and complex data types

- gold Open Access which fosters wider collaboration and increased citations

- maximum visibility for your research: over 100M website views per year

At $B M C$, research is always in progress.

Learn more biomedcentral.com/submissions 